Análisis espacial de las condiciones laborales de la mujer en el sector agropecuario en México

Jorge López-Martínez y Alejandro Molina-Vargas 
Lecturas de Economía, 91 (julio-diciembre 2019), pp. 181-209

Jorge López-Martinezy Alejandro Molina-Vargas

\section{Análisis espacial de las condiciones laborales de la mujer en el sector agropecuario en México}

Resumen: El objetivo del presente trabajo es medir los cambios espaciales de las condiciones laborales de la mujer en el sector agropecuario en las 32 entidades federativas de México, durante el periodo 2008-2017. Para ello, se elabora un índice que considera tres factores: tasa de población femenina ocupada que percibe alguna remuneración, tasa de población femenina ocupada que tiene acceso a saludy otras prestaciones, y tasa de población femenina ocupada que no recibe ninguna remuneración. Los resultados del indice para 2017 muestran una persistente precariedad en las condiciones laborales de la mujer en los estados del sur: Guerrero, Oaxaca y Chiapas, con indices de 0,13, 0,17 y 0,2 respectivamente. En contraste, los estados del noroeste: Baja California, Sinaloa, Sonora, Baja California Sury Chibuahua, continúan presentando los mejores resultados con indices de 0,74, 0,66, 0,66, 0,59 y 0,55 respectivamente. Estados relativamente más industrializados, como Querétaro, Aguascalientes, Guanajuato, Coabuila y Nuevo León, han mejorado las condiciones laborales al incrementarse el indice de manera considerable, lo que sugiere la existencia de un efecto spillover sobre el sector agropecuario.

Palabras clave: mujeres; condiciones laborales; sector agropecuario; espacialidad.

Clasificación JEL: J16, J43, R12.

\section{Spatial analysis of working conditions for women in the agricultural sector in Mexico}

Abstract: The objective of this paper is to measure the spatial changes that have occurred in the working conditions of women in the agricultural sector in the 32 states of Mexico, during the 2008-2017 period. For this, an index is elaborated that considers three factors: rate of occupied female population that receives some remuneration, rate of occupied female population that has access to bealth and other benefits, and rate of employed female population that does not receive any remuneration. The results of the index for 2017 show persistent precariousness in the working conditions of women in the southern states: Guerrero, Oaxaca and Chiapas, with indexes of 0.13, 0.17 and 0.2, respectively. In contrast, the states of the northwest: Baja California, Sinaloa, Sonora, Baja California Sur and Chibuabua, continue presenting the best results with indexes of $0.74,0.66,0.66,0.59$ and 0.55 , respectively. Relatively more industrialized states, such as Querétaro, Aguascalientes, Guanajuato, Coabuila and Nuevo León, have improved working conditions by increasing the index considerably, suggesting the existence of a spillover effect on the agricultural sector.

Keywords: women; working conditions; agricultural sector; spatiality.

JEL Classification: J16, J43, R12.

\section{Analyse spatiale des conditions de travail des femmes dans le secteur agricole au Mexique}

Résumé: L'objectif de ce travail est de mesurer les changements spatiaux des conditions de travail des femmes dans le secteur agricole dans les 32 États fédératifs du Mexique, au cours de la période 2008-2017. Pour cela, un indice est construit afin de prend en compte trois facteurs: le taux de population féminine occupée qui reçoit une certaine rémunération, le taux de population féminine occupée qui a accès à la santé et d'autres avantages, et le taux de population féminine occupée qui ne reçoit aucune rémunération. Les résultats de l'indice pour 2017 révèlent une précarité persistante dans les conditions de travail des femmes dans les États du sud: Guerrero, Oaxaca et Chiapas, avec des indices de 0,13, 0,17 et 0,2 respectivement. En revanche, les États du nord-ouest: Basse-Californie, Sinaloa, Sonora, Basse-Californie du Sud et Chihuahua continuent à présenter les meilleurs résultats avec des indices de 0,74, 0,66, 0,66, 0,59 et 0,55 respectivement. Des États plus industrialisés, tels que Querétaro, Aguascalientes, Guanajuato, Coahuila et Nuevo León, ont amélioré les conditions de travail des femmes en augmentant considérablement l'indice, ce qui suggère l'existence d'un effet spillover dans le secteur agricole.

Mots clés: femmes; conditions de travail; secteur agricole; spatialité

Classification JEL: J16, J43, R12. 


\title{
Análisis espacial de las condiciones laborales de la mujer en el sector agropecuario en México
}

\author{
Jorge López-Martínez $\mathbb{1}^{\mathrm{a}}$ y Alejandro Molina-Vargas $\mathbb{C}^{\mathrm{b}}$
}

-Introducción. -I. Participación laboral de la mujer: una revisión de la literatura. -II. El sector agropecuario en los estados mexicanos. -III. Distribución laboral de la mujer en el sector agropecuario. -IV. Índice de condiciones laborales. -Conclusiones. -Referencias.

doi: 10.17533/udea.le.n91a06

Primera versión recibida el 12 de septiembre de 2018; versión final aprobada el 30 de enero de 2019

\section{Introducción}

El empleo es una de las variables más importantes en los objetivos de política económica; el crecimiento del número de empleos, es un indicador de que la economía va bien. Sin embargo, en la última década se ha puesto mayor atención a la calidad del trabajo. Según datos del Instituto Nacional de Estadística y Geografía (INEGI), en 2017 la población mexicana ascendió a 123.98 millones de personas, en los últimos 12 años la tasa promedio de crecimiento ha sido de 1,53\%. Del total de mexicanos, el 51,67\% corresponde a la población femenina. De otra parte, en el mismo periodo, la población económicamente activa (PEA) alcanzó los 54,7 millones de personas, a un ritmo de crecimiento promedio anual de 1,98\%. Esto quiere decir que cada vez es mayor el número de personas que entran al rango de edad de trabajar y buscan trabajo. Al primer trimestre de 2017, por ejemplo, 51,86 millones de personas estaban ocupadas.

En este estudio se analizan los cambios en las condiciones laborales de las mujeres en el sector agropecuario mexicano, analizados por estados. En

a Jorge López-Martinez: profesor de la Facultad de Estudios Superiores-Acatlán, Universidad Nacional Autónoma de México, México. Dirección postal: 53150. Dirección electrónica: j_lopez@comunidad.unam.mx. https://orcid.org/0000-0001-5656-9923

b Alejandro Molina-Vargas: profesor investigador de la Universidad Politécnica Metropolitana de Hidalgo, México. Dirección postal: 43860. Dirección electrónica: amolina@upmh.edu.mx. http:/ / orcid.org/0000-0001-9319-4913 
López-Martínez y Molina-Vargas: Análisis espacial de las condiciones laborales de la mujer...

general, la creciente participación de la mujer en el mercado laboral se ha dado con un mayor nivel de instrucción, lo que ha sido tema de análisis en la literatura. Tan solo en la primera década del siglo XXI, la mitad de la matrícula estudiantil universitaria en México correspondía al sexo femenino (De Garay, 2013). Sin embargo, a pesar de que en la actualidad las mujeres cuentan con una mejor preparación académica, esto no se ha visto reflejado en términos reales en sus percepciones salariales. En este contexto, es especialmente la mujer quien enfrenta un entorno adverso en el sector agropecuario, en el cual el mercado laboral presenta un esquema de segmentación de salarios que no corresponde a la productividad que el nivel de educación genera (López \& Molina, 2018). Además, se trata de un sector que se caracteriza, en su mayor parte, por ser de temporada; es decir, la siembra y la cosecha son factores estacionales que imprimen esta naturaleza a los contratos laborales.

El presente trabajo tiene como objetivo medir los cambios en las condiciones laborales de la mujer en el sector agropecuario, en las 32 entidades federativas de México, durante el periodo 2008-2017. Para ello, se elabora una serie de mapas que muestran la distribución espacial de factores como: población ocupada no remunerada, población ocupada cuya remuneración es superior a cinco salarios mínimos, población ocupada que tiene acceso a salud y otras prestaciones, y población ocupada que tiene un nivel de instrucción de media superior y superior. Aunado a esto, se construye un índice que permite medir los cambios en las condiciones laborales y que toma en cuenta tres factores: tasa de población femenina ocupada que percibe alguna remuneración (ponderada por segmentos de ingresos medidos en salarios mínimos), tasa de población femenina ocupada que tiene acceso a salud y otras prestaciones, y tasa de población femenina ocupada que no recibe ninguna remuneración. La base de datos utilizada la proporciona el INEGI, a través de la Encuesta Nacional de Ocupación y Empleo (ENOE). Por la disponibilidad de datos, el análisis se limita al periodo 2008-2017.

El presente trabajo se estructura de la siguiente manera: después de la introducción, en la primera parte, se hace la revisión de la literatura; en la segunda, se analizan las características de la producción agropecuaria en los estados; en la tercera parte, se observa la participación de la mujer y su situación laboral en el sector por estados mediante la técnica del mapeo; en 
la cuarta parte se construye el índice de condiciones laborales que mide los cambios presentados; y, por último, se recogen las conclusiones.

\section{Participación laboral de la mujer: una revisión de la literatura}

La participación laboral de la mujer en la economía ha sido de amplio interés en la literatura académica y se ha estudiado desde diferentes enfoques. Por ejemplo, se han realizado trabajos conceptuales para incorporar la perspectiva de género en el análisis económico (Ferber \& Nelson, 1993; Solow, 1993; Castaño, 1999); trabajos formales que incorporan una distinción de género y ofrecen nuevos elementos para la construcción de la teórica microeconómica, es decir, distinguen la conducta económica de hombres y mujeres (Noriega, 2010; Villegas, 2012); e investigaciones donde, desde una visión macroeconómica, se destaca la contribución de la mujer y los beneficios macroeconómicos que se obtienen al incorporar la equidad de género. Sin embargo, los estudios señalan que, en su mayoría, la participación laboral de las mujeres es no remunerada, o en su defecto, cuando se les remunera, es porque se incorporan al sector informal (Elborgh et al., 2013).

Así mismo, Craig, Garnsey y Rubery (1985) analizan el papel de la mujer en el mercado laboral bajo el enfoque de la teoría de la segmentación del mercado de trabajo. Esta postura consiste en que el mercado laboral se divide en segmentos que presentan mecanismos de asignación salarial diferentes y en la inexistencia de movilidad entre segmentos (Doeringer \& Piore, 1971; Piore, 1972). Dentro de la segmentación del mercado laboral, destaca la visión institucionalista, en la cual el mercado laboral se divide en dos segmentos: uno primario, donde se ofrecen empleos con salarios relativamente altos, buenas condiciones de trabajo, posibilidades de ascenso, equidad y debido proceso en la administración de las normas de trabajo y, sobre todo, estabilidad laboral; y el segmento secundario, donde se ofrecen empleos con salarios bajos, malas condiciones de trabajo, pocas posibilidades de progreso, inestabilidad y alta rotación (Piore,1972).

Desde otra perspectiva, Becker (1981) considera, bajo el enfoque de la teoría del capital humano, que la participación de la mujer en el trabajo remunerado estará determinada por el número de hijos que tenga, la 
López-Martínez y Molina-Vargas: Análisis espacial de las condiciones laborales de la mujer...

preparación académica, el salario potencial y por el total del ingreso de la familia.

A nivel agregado, en la literatura empírica, sobre el mercado laboral mexicano, se destacan aspectos como el comportamiento del empleo femenino durante periodos recesivos en el marco del ciclo económico (Cervini, 2000). Así mismo, se pone énfasis en las desigualdades entre los sexos, la discriminación en el trabajo, las diferencias salariales en el mercado laboral entre sexos, la pobreza y marginación, la precariedad laboral de la mujer, a pesar de que su participación cada vez tiene un mayor nivel académico (Martínez, Quintero \& Viianto, 2015; Mendoza, Cardero \& Ortiz, 2017). Las desigualdades entre hombres y mujeres respecto a sus oportunidades laborales ponen a la mujer en una situación más precaria respecto al hombre, lo que amplía las brechas en las tasas de participación y de desempleo entre los dos sexos (Martínez et al., 2015). Por otro lado, Mendoza et al. (2017) muestran que, en el ingreso laboral mexicano, los hombres ganan más que las mujeres, a pesar de que se incluye la dotación de capital humano y capacitación. Los autores concluyen que las tendencias de los diferenciales del ingreso de largo plazo se explican, principalmente, por el comportamiento de la discriminación salarial y las dotaciones por años de experiencia.

A nivel sectorial, la participación laboral de la mujer en la industria y en las maquilas en la economía mexicana ha sido de amplio interés (Gabayet \& Lailson, 1990; Cordourier \& Gómez, 2004; Carrillo \& Hernández, 2014). Los estudios evalúan aspectos como la composición de la fuerza laboral, las condiciones de trabajo, los salarios bajos. Cabe resaltar que una de las características de las maquilas es emplear, en mayor proporción, la fuerza de trabajo femenino, por su esmero, paciencia y disciplina; además las emplean con salarios menores.

En el sector servicios, los trabajos de Guzmán (2004), Moctezuma, Narro y Orozco (2014) y García (2015) muestran la situación inequitativa y desfavorable de la mujer, pues la diferencia de ingresos respecto a los hombres se da en casi todos los sectores de la economía. Así se resalta también en el estudio que llevó a cabo Arragiada (1990) en cinco ciudades de América Latina, en el cual se evidencia que, a pesar de tener el mismo 
nivel de instrucción, el ingreso medio de los hombres y mujeres ocupados presenta grandes diferencias. Adicionalmente, mientras mayor es el nivel de instrucción, también es mayor la diferencia de ingresos, en todos los casos en deterioro de las mujeres. Algunos estudios muestran que solo en el mercado laboral académico es donde existen las mismas condiciones laborales y de ingresos para ambos géneros (De Garay, 2013). En esta misma línea, Flores y Salas (2015) señalan que la variable más significativa es la educación superior, ya que a partir de los estudios profesionales se comienzan a invertir las brechas de género, y con estudios de posgrado, la mujer obtiene mejores condiciones laborales que el hombre.

En cuanto al mercado de trabajo agrícola, este se caracteriza por la preferencia a contratar trabajadores hombres y por ser el sector que presenta la menor calidad del empleo (Hernández, 2014; Flores \& Salas, 2015). Según Hernández (2014), las mujeres se convierten en un componente residual en este mercado, pues su ocupación se encuentra fuertemente vinculada a los ciclos agrícolas; es decir, tienden a encontrarse ocupadas preferentemente en los picos de demanda, pero a ser desempleadas en las etapas iniciales y finales del ciclo agrícola. Por su parte, Caro (2012) destaca que también la estabilidad del salario y del empleo, como se conoce en la sociedad, ha motivado la inestabilidad en los ingresos con la generalización del empleo precario. Esto se hace más evidente en el mercado laboral agrícola de Chile, donde existen deficiencias en el funcionamiento del mercado de trabajo y de las instituciones laborales, lo que afectan especialmente a los trabajadores temporales. Aun cuando se trata de un sector que ha aumentado su productividad, no ha logrado traspasar dicho incremento a los salarios, ni conseguir que el crecimiento económico se traduzca en el mejoramiento de la calidad de los empleos, como podría esperarse. En este mismo sentido, López y Molina (2018) muestran que, para el caso de México, a pesar de que hay un ligero aumento en la productividad, no se ha reflejado en el salario. Igualmente, revelan que el mayor grado de escolaridad que presenta la mujer no ha contribuido a que acceda en igual medida al segmento de mayores ingresos; por el contrario, ha provocado una presión a la baja sobre los salarios, de tal manera que para mantener o mejorar su ingreso ha sido necesario que tengan un mayor número de horas trabajadas. 
López-Martínez y Molina-Vargas: Análisis espacial de las condiciones laborales de la mujer...

En un estudio sobre el trabajo de las mujeres en las zonas rurales de América Latina, se reconoce que las mujeres de estas zonas, en particular, suelen trabajar en actividades no remuneradas, estacionales y en empresas familiares (Wainerman \& Recchini, 1981, citados por Arriagada, 1990). En esta misma línea, Valenciano, Capobianco y Uribe (2017) señalan que las mujeres rurales son uno de los grupos sociales más vulnerables en el ámbito laboral latinoamericano, debido a su baja participación laboral y a que, en la categorización ocupacional se las clasifica, mayoritariamente, como trabajadoras por cuenta propia y sin remuneración. Lahoz (2011) analiza los programas implementados por el Gobierno mexicano para apoyar a la mujer campesina y concluye que, a pesar de estos, la situación laboral de la mujer en el sector rural sigue siendo desventajosa. Además, reconoce que el papel que ellas desempeñan es de vital importancia para el sistema alimentario, el cual comprende vínculos como la producción, distribución y consumo de alimentos.

\section{El sector agropecuario en los estados mexicanos}

En promedio, de 2008 a 2017, el sector agropecuario participó en el Producto Interno Bruto (PIB) total con un 3,2\%, mientras que los sectores secundario y terciario lo hicieron con un 35,3 y $61,4 \%$, aproximadamente. En la literatura se sostiene que, conforme se da el desarrollo económico de los países, estos transitan del sector primario al terciario. Según datos del Banco Mundial, en países desarrollados como Alemania y Estados Unidos el sector agropecuario aporta alrededor del 0,9 y 1,43\% del total del PIB, respectivamente, mientras que en países como Argentina, Brasil y Chile contribuye con el 7,95\%, 5,5\% y 4,3\%, respectivamente. En este contexto, el crecimiento promedio anual del sector en el periodo analizado fue de 1,48 \%, apenas compatible con el ritmo de crecimiento de la población, que se ubica en $1,3 \%$, pero insuficiente para alcanzar una proporción aceptable respecto al total de la economía.

Para 2008, la producción agropecuaria se concentró en ocho estados: Jalisco, Sinaloa, Michoacán, Veracruz, Sonora, Chihuahua, Guanajuato y Puebla, los cuales, en conjunto, proveyeron poco más del $50 \%$ del total 
de la producción. En contraste, la Ciudad de México, Quintana Roo, Baja California Sur, Campeche y Tlaxcala aportaron cada una de ellas menos de $1 \%$, y en conjunto apenas el 2,62\%. Para 2017, respecto a la contribución porcentual que realizaron las entidades federativas al total de la producción agropecuaria nacional, no se observan cambios significativos: las ocho entidades federativas ya señaladas son las que mayor contribución hicieron al PIB agropecuario nacional (Figura 1), mientras que estados con superficies extensas como Quinta Roo, Baja California Sur y Campeche, no presentan mayor actividad agropecuaria, ya que están orientadas al turismo o al sector energético. En los casos de Quintana Roo y Campeche, la mayor parte de su extensión territorial corresponde a selva subcaducifolia, lo que no permite la actividad agropecuaria, mientras que el territorio de Baja California Sur es en su inmensa mayoría de matorral xerófilo.

En la Tabla 1 se destaca la vocación agropecuaria de estos estados. Cabe mencionar que, en casos como el de Sonora y Michoacán, la producción está altamente ligada a la exportación, lo que explica, como veremos adelante, las características distintivas que tienen estos estados respecto a la ocupación femenina.

En 2008, 741 mil mujeres estuvieron ocupadas en el sector agropecuario, y la mitad de este total se concentró en cinco estados: Puebla, Oaxaca, Guerrero, Veracruz e Hidalgo (Figura 2). Trece estados contribuyeron con menos del $1 \%$, por lo que, en conjunto, apenas sumaron un 7,79\% del total de población femenina ocupada en este sector. Por otro lado, para 2016, el total de mujeres ocupadas en el mismo sector fue de 718 mil, es decir, hubo un retroceso del $3 \%$. En seis estados se concentró la mitad: continuaron los mismos tres primeros estados de 2008, aquellos que más absorben trabajo femenino y en los que el trabajo es no remunerado, mientras que Jalisco, Guanajuato y Michoacán se posicionaron entre los estados que ocupan más mujeres en el sector. Finalmente, once estados contribuyeron de manera individual con menos del $1 \%$, y en total sumaron 6,5\% (Figura 2). 
López-Martínez y Molina-Vargas: Análisis espacial de las condiciones laborales de la mujer...

Tabla 1. Producción agropecuaria de los principales estados

\begin{tabular}{l|l|c|l|c}
\hline & \multicolumn{2}{|c|}{2008} & \multicolumn{2}{c}{2017} \\
\hline Estado & Producto & $\begin{array}{c}\text { \% del valor de } \\
\text { la producción* }\end{array}$ & Producto & $\begin{array}{c}\text { \% del valor de } \\
\text { la producción* }\end{array}$ \\
\hline Jalisco & Huevo para plato & 36,1 & Huevo para plato & 33,2 \\
Sinaloa & Maíz grano blanco & 46,2 & Maíz grano blanco & 47,8 \\
Michoacán & Aguacate Hass & 40,2 & Aguacate Hass & 39,2 \\
Veracruz & Carne de bovino & 40,0 & Carne de bovino & 42,8 \\
Sonora & Carne de porcino & 55,0 & Carne de porcino & 47,2 \\
Chihuahua & Carne de bovino & 35,2 & Carne de bovino & 47,8 \\
Guanajuato & Carne de ave & 30,5 & Carne de ave & 29,3 \\
Puebla & Huevo para plato & 35,4 & Huevo para plato & 36,9 \\
\hline
\end{tabular}

Nota: ${ }^{*}$ en el caso de huevo para plato, carne de bovino, carne de porcino y carne de ave se refiere a la producción pecuaria del estado en cuestión. En el caso del maiz grano blanco y aguacate Hass, se refiere a la producción agrícola del estado en cuestión.

Fuente: elaboración propia con base en datos de la Oficina Estatal de Información para el Desarrollo Rural Sustentable (OEIDRUS), Infografía Alimentaria (SIAP).

En conjunto, se observa una disociación entre aquellas entidades que más aportan a la generación de la producción agropecuaria y aquellas que tienen una mayor ocupación: las entidades que mayor producción generan durante el periodo no son las que ocupan el mayor número de mujeres, a excepción de Michoacán que en 2016 fue el segundo mayor productor agropecuario y el sexto en ocupación femenina. Además, mientras que el sector creció en $15,45 \%$ durante el periodo, la ocupación femenina decreció en $3 \%{ }^{1}$ En este sentido, la ley de Okun no se cumple, pues no existe una relación causal definida entre el nivel de producción y la ocupación femenina (López \& Molina, 2018). Esto confirma que, como señala Hernández (2014), las mujeres se han convertido en un componente residual en el mercado laboral agrícola.

1 Según datos de la ENOE, durante el periodo, la ocupación total creció un 9,76\%, mientras que la masculina lo hizo en un 11,53\% y desplazó a la femenina. De otro lado, mientras que en 2008 las mujeres ocupadas representaron el 12,11\% del total de ocupados, para 2016 solo representaron el 10,7\%. 
Figura 1. Participación porcentual al PIB agropecuario nacional por entidad federativa

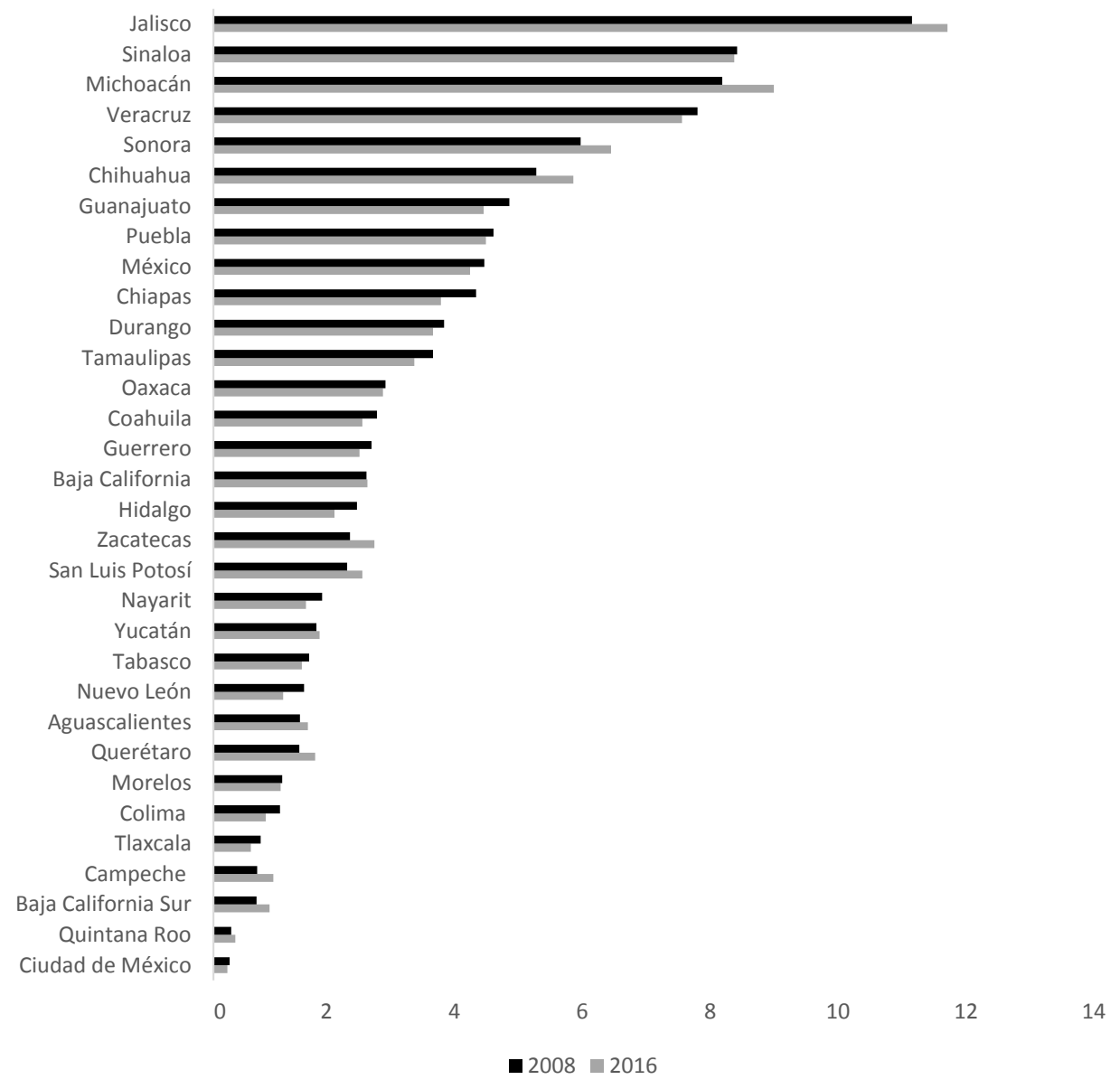

Fuente: elaboración propia con base en datos del INEGI.

En las Tablas 2 y 3, se presentan las correlaciones de Pearson entre la producción agropecuaria de cada estado y su respectiva ocupación femenina en el sector, para 2008 y 2016. 
López-Martínez y Molina-Vargas: Análisis espacial de las condiciones laborales de la mujer...

Figura 2. Participación porcentual de la ocupación femenina en el sector agropecuario

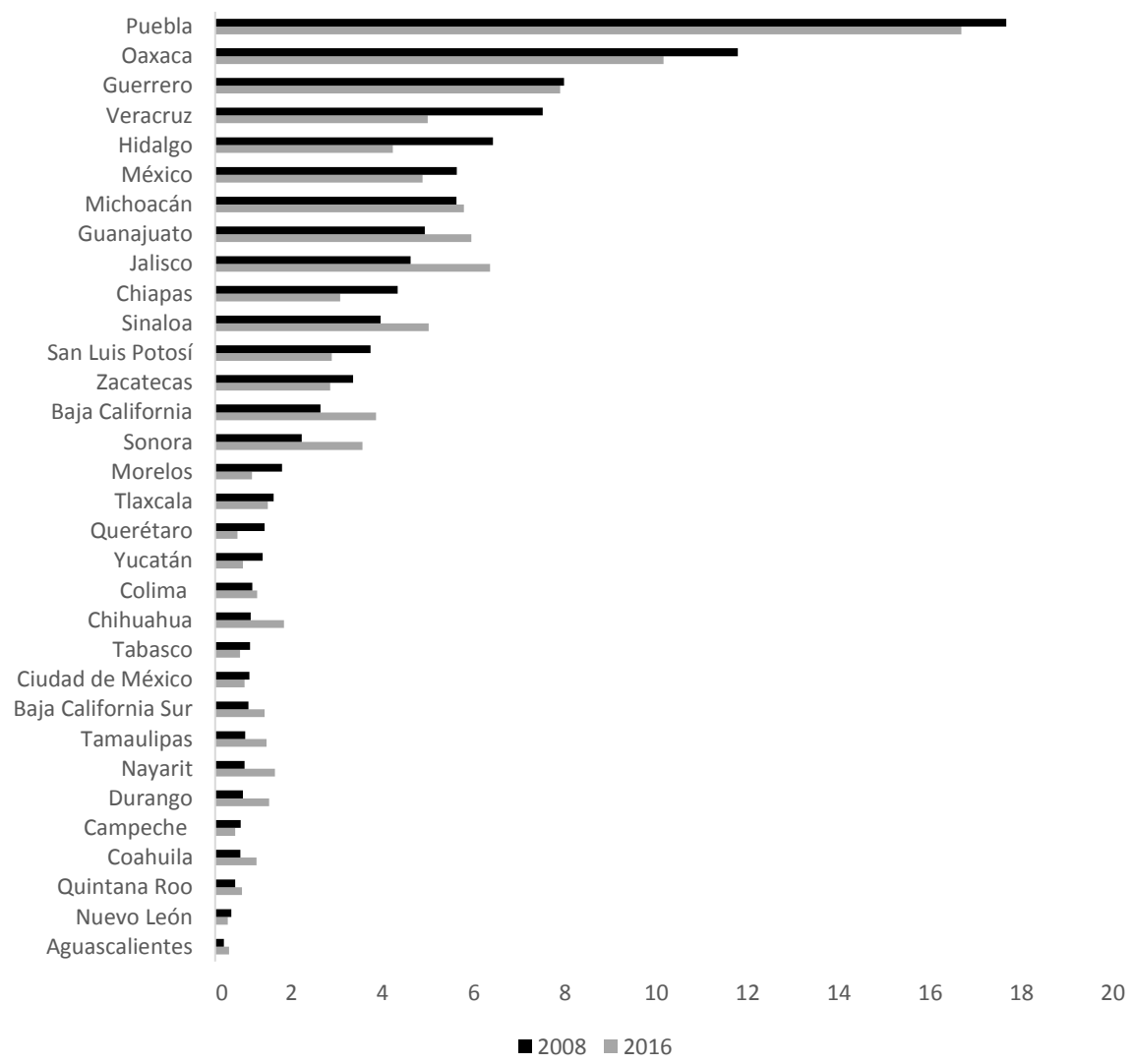

Fuente: elaboración propia con base en datos del INEGI.

Tabla 2. Correlación entre el PIB agropecuario y el empleo femenino, 2008

\begin{tabular}{lcc}
\hline & Ocupación femenina (\%) & PIB agropecuario (\%) \\
\hline Ocupación femenina (\%) & 1,000000 & 0,473308 \\
PIB agropecuario (\%) & 0,473308 & 1,000000 \\
\hline
\end{tabular}

Fuente: elaboración propia con base en datos del INEGI. 
Tabla 3. Correlación del PIB agropecuario y el empleo femenino, 2016

\begin{tabular}{ccc}
\hline & Ocupación femenina (\%) & PIB agropecuario (\%) \\
\hline Ocupación femenina (\%) & 1,000000 & 0,459873 \\
PIB agropecuario (\%) & 0,459873 & 1,000000 \\
\hline
\end{tabular}

Fuente: elaboración propia con base en datos del INEGI.

El valor de la correlación de Pearson para 2008 es de 0,4733, mientras que para 2016 es de $0,4598 .^{2}$ Se evidencia entonces que, para el 2016, el grado de asociación entre la producción agropecuaria y el empleo femenino en cada uno de los estados del país es menor. El valor de correlación de Pearson se explica, sobre todo, por la disociación que existe entre producción agropecuaria y ocupación femenina en estados como Puebla, Oaxaca y Guerrero, donde la contribución al PIB agropecuario es baja, mientras que la ocupación (no remunerada) es relativamente alta.

\section{Distribución laboral de la mujer en el sector agropecuario}

La distribución laboral de la mujer en el sector agropecuario presenta características importantes a nivel estatal. Este análisis se presenta a través de la generación de mapas por estados federativos en los siguientes rubros: ocupación total femenina, ocupadas no remunerada, ocupadas que perciben más de cinco salarios mínimos, ocupadas que tienen acceso a salud y otras prestaciones, y finalmente, ocupadas que tienen un nivel de instrucción mediasuperior y superior. Cada rubro está en términos porcentuales respecto al total estatal, a excepción de la ocupación total que esta referenciada con el total nacional.

Anne Fouquet (2005) divide en ocho zonas económicas a México: Noroeste (Baja California, Baja California Sur, Chihuahua, Durango, Sinaloa y Sonora), Noreste (Coahuila, Nuevo León y Tamaulipas), Occidente (Colima, Jalisco, Michoacán y Nayarit), Oriente (Hidalgo, Puebla, Tlaxcala y Veracruz),

2 El coeficiente de correlación de Pearson toma valores que van de -1 a 1. Los valores negativos indican una relación inversa entre las variables, el 0 indica que no hay asociación alguna, mientras que los valores positivos indican una relación directa. 
López-Martínez y Molina-Vargas: Análisis espacial de las condiciones laborales de la mujer...

Centro-Norte (Aguascalientes, Guanajuato, Querétaro, San Luis Potosí y Zacatecas), Centro-Sur (Ciudad de México, Estado de México y Morelos), Suroeste (Chiapas, Guerrero y Oaxaca) y Sureste (Campeche, Quintana Roo, Tabasco y Yucatán), lo que permite, por un lado, observar la situación particular de cada una de las entidades federativas en el marco de las zonas económicas y así tener un panorama completo de las condiciones laborales de las mujeres en el sector; $y$, por otro lado, permite la elaboración de políticas públicas diseñadas para hacer frente a cada problemática que presenta cada entidad o zona.

La Figura 3 contiene la distribución porcentual de la ocupación femenina en el sector agropecuario por entidad federativa para el primer trimestre de 2008 y el primero de $2017 .{ }^{3}$ Del total de mujeres ocupadas en el país, se observa su distribución porcentual en los distintos estados de la república, sin tomar en cuenta ninguna de las otras variables que caracterizan el tipo de ocupación, de tal manera que esta variable representa el universo de cada entidad federativa para el análisis posterior.

En la participación porcentual de cada entidad federativa en el nivel de ocupación femenina nacional, se observan algunos cambios significativos de 2008 a 2017. El bajo ritmo de crecimiento del sector $(1,48 \%$ en promedio anual) estuvo acompañado de una reconfiguración porcentual en la ocupación. Para 2008, el grueso de la ocupación femenina se concentró en algunos estados de las zonas oriente y suroeste, especialmente Puebla, Veracruz y Oaxaca. Se observa una franja con niveles de ocupación intermedia que comprende algunos estados de las zonas centro-norte, centro-sur, suroeste y occidente (San Luis Potosí, Estado de México, Guerrero, Chiapas, Michoacán y Jalisco). Finalmente, a excepción de Sinaloa, las regiones noroeste y noreste del país presenta porcentajes mínimos de ocupación femenina. Para 2017 se observa una estructura porcentual distinta, pues la brecha de ocupación entre las zonas del norte y del sur se redujo ligeramente. Si bien los estados de las zonas oriente y suroeste continúan teniendo

3 En las actividades del sector agropecuario, el componente estacional es una parte importante de las series de datos, para evitar los sesgos provenientes de esta parte de la serie temporal, se trabajó con datos referentes al primer trimestre al inicio del periodo contra el primer trimestre del término del periodo. 
porcentajes altos de ocupación, las zonas noroeste y occidente absorben un mayor porcentaje de ocupación femenina en relación con 2008; los casos más importantes son los estados de Sonora y Baja California, que en términos porcentuales pasaron de 2,44 a 4,76\% y de 1,7 a 3,26\% respectivamente. En los últimos años, Sonora ha tenido un mayor desempeño en la actividad agropecuaria. Esto lo ha convertido en uno de los principales exportadores de productos agropecuarios del país, donde se destaca con productos como en el trigo, la uva, los espárragos, la papa y la carne de cerdo, (Infografía Alimentaria de la Secretaría de Agricultura, Ganadería Desarrollo Rural, Pesca y Alimentación -SAGARPA-). En las zonas oriente y suroeste, a excepción de Puebla que pasó de una ocupación de 15,79 en 2008 a 18,15 en 2017, el resto de las entidades bajó su participación en la ocupación, por ejemplo, Oaxaca, que en 2008 tuvo un $11 \%$, en 2017 fue de 8,11\%.

Figura 3. Distribución porcentual de la ocupación femenina

Primer trimestre de 2008

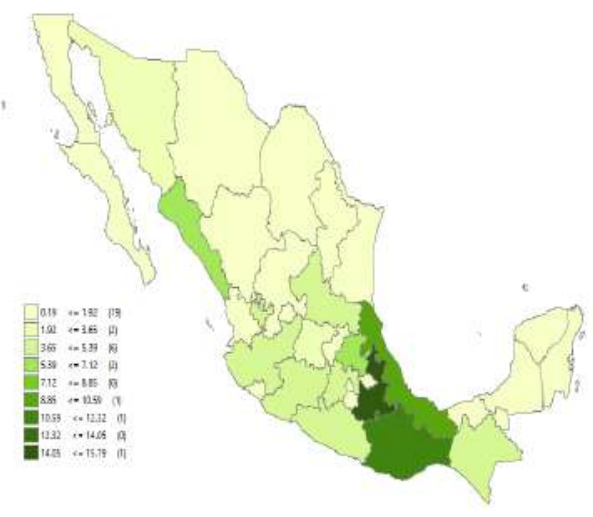

Primer trimestre de 2017

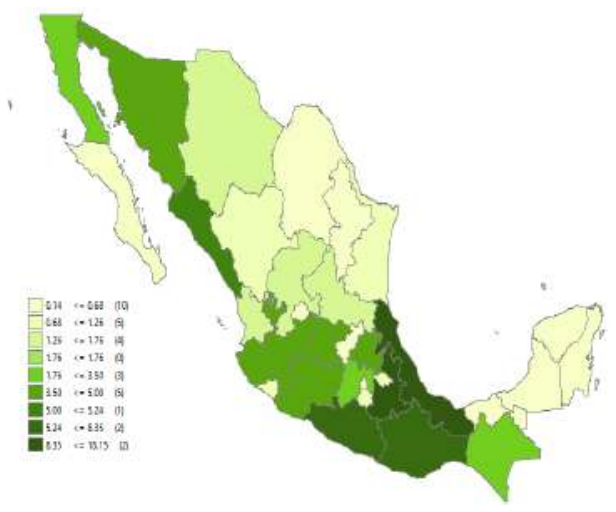

Fuente: elaboración propia con base en datos de la ENOE y el INEGI.

Una de las características principales de la ocupación es la remuneración. En el sector agropecuario, en la mayoría de los casos de Puebla, Oaxaca y Guerrero, las mujeres no perciben remuneración alguna por su trabajo. En términos porcentuales por entidad federativa, de 2008 a 2017, se observan algunos cambios que, en general, representan un retroceso (Figura 4). 
López-Martínez y Molina-Vargas: Análisis espacial de las condiciones laborales de la mujer...

Tales son los casos de Chihuahua, Tamaulipas, Durango, Jalisco, Veracruz, Campeche, Yucatán y Quintana Roo, donde el porcentaje de mujeres que no perciben ningún tipo de ingreso por su trabajo respecto al total de ocupación por entidad federativa se incrementó. Por otro lado, se observan menores porcentajes de mujeres no remuneradas en Sonora, Baja California Sur, Coahuila, Estado de México, Hidalgo y Oaxaca. Lo que evidencia este comportamiento es la persistencia del empleo no remunerado sobre la corta temporalidad del empleo remunerado. De esta manera, cuando la ocupación total cae, se trata, en su mayoría de empleo remunerado, por lo que su contribución porcentual al total de empleo disminuye de manera significativa y, por el contrario, el porcentaje del empleo no remunerado se incrementa más que proporcionalmente. Por otro lado, continúa presentándose una gran brecha entre el norte y el sur: mientras que, en promedio, en las zonas noreste y noroeste en 2008 el 19,65\% de mujeres empleadas no percibían ingreso por su trabajo, en la zona oriente y suroeste el promedio fue de 65,13\%. Para 2017, las cifras fueron 15,12 y $57,9 \%$, respectivamente. Si bien, se evidencia que la brecha se cerró ligeramente, varios estados presentaron un retroceso en el porcentaje de las mujeres que percibieron alguna remuneración por su trabajo. Si asociamos la Figura 3 con la Figura 4, en la cual se ilustra el porcentaje de mujeres no remuneradas respecto al total de mujeres ocupadas por entidad federativa, podemos ver que varios estados que presentaron una mayor tasa de ocupación femenina no remunerada, también presentaron una tasa de ocupación mayor respecto al inicio del estudio. Tal es el caso de Chihuahua, donde el número de mujeres ocupadas pasó de 4,784 en 2008 a 12,379 en 2017, mientras que el porcentaje de mujeres no remuneradas creció de 12,21 a $16,14 \%$; es decir, en muchos casos las mujeres comenzaron a trabajar en el sector, pero la mayor parte de su trabajo sigue sin ser remunerado.

En el otro extremo del segmento de remuneraciones, tenemos aquellas mujeres que perciben un ingreso mayor a cinco salarios mínimos. La ocupación con este tipo de remuneración se encuentra en el sector agropecuario que está ligado a la agroindustria, ${ }^{4}$ la cual demanda de éste su materia prima. La Figura 5 muestra el porcentaje de mujeres que obtuvieron

4 Cabe aclarar que la agroindustria no forma parte del sector agropecuario, sino del sector manufacturero. La agroindustria la comprenden los subsectores de alimentos, y bebidas y 
más de cinco salarios mínimos por entidad federativa-respecto del total de ocupación femenina del sector agropecuario por entidad federativa-. Se observa que la mayoría de los estados del centro sur, suroeste y sureste continúan presentando porcentajes muy bajos de mujeres que perciben este nivel salarial. En contraste, los estados del noroeste, como Sonora, Chihuahua y Sinaloa, y Tamaulipas en el noreste, continuaron con tasas altas. Por otro lado, algunos estados cambiaron su tendencia, como el caso de Campeche y Guanajuato, cuyas tasas cayeron, mientras que Baja California, Nayarit, Jalisco e Hidalgo presentaron una evolución favorable. En total, el sector agroindustrial tuvo un crecimiento promedio anual de 1,34\% de 2008 a 2013, y de 1,8\% de 2013 a 2017(SAGARPA, 2017), lo que sin duda es un factor positivo en cuanto a las remuneraciones superiores a cinco salarios mínimos, debido a la estrecha relación que el crecimiento guarda con el sector agropecuario, principalmente en el noroeste del país.

Figura 4. Porcentaje de mujeres no remuneradas respecto al total de mujeres ocupadas

Primer trimestre de 2008

Primer trimestre de 2017
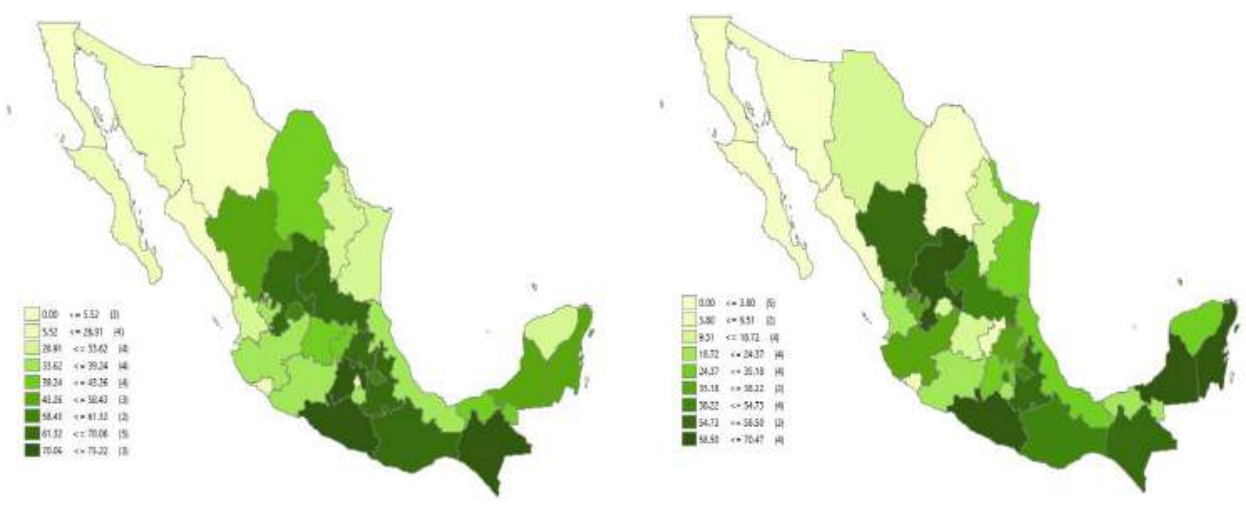

Fuente: elaboración propia con base en datos de la ENOE y el INEGI.

tabaco. De estos dos subsectores, el de alimentos comprende alrededor de tres cuartas partes (SAGARPA, 2013). 
López-Martínez y Molina-Vargas: Análisis espacial de las condiciones laborales de la mujer...

Figura 5. Porcentaje de mujeres que perciben más de cinco salarios minimos respecto al total de mujeres ocupadas
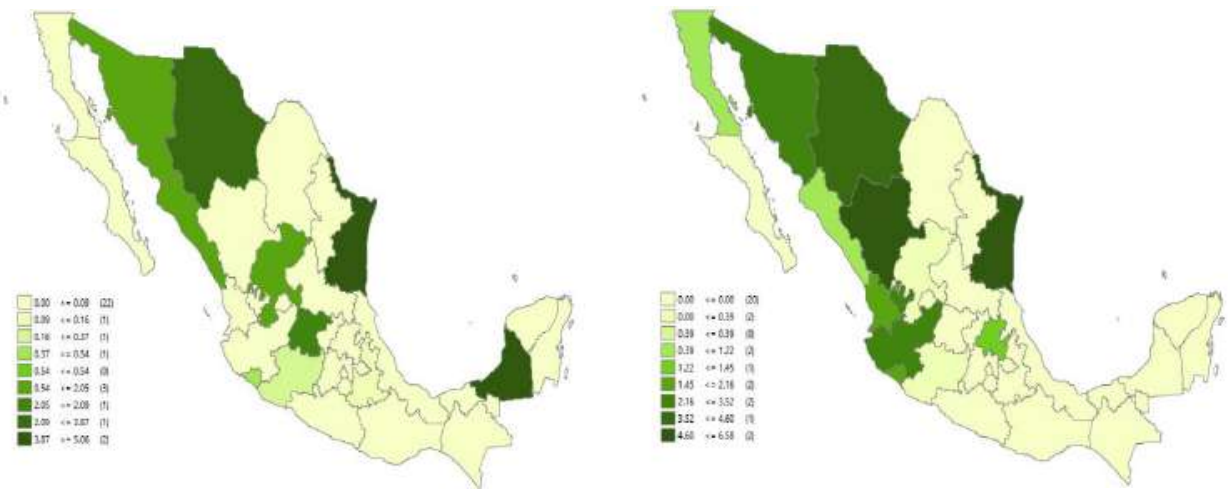

Fuente: elaboración propia con base en datos de la ENOE y el INEGI.

En cuanto al acceso a salud y otras prestaciones, se observa que, con algunos pequeños contrastes, la distribución es muy similar al inicio y al final del periodo. En los estados del noroeste, noreste y occidente se tienen mejores condiciones relativas de trabajo femenino. Como señalan Aranda y Castro (2016), es tal la absorción de mano de obra femenina, que existe una migración anual de mujeres que se trasladan de los estados sureños a las regiones donde se practican cultivos intensivos de productos como la uva y el jitomate, principalmente en los estados de Baja California, Sonora y Sinaloa. Aunque en estas regiones aún se puede mejorar en factores que se traduzcan en condiciones idóneas de trabajo para las mujeres dentro del sector, es precisamente aquí donde se presentan las tasas más altas de mujeres que tienen acceso a salud y otras prestaciones. En contraposición, nuevamente es en los estados del centro sur (exceptuando a Morelos), suroeste, sureste y occidente donde los porcentajes son menores. Si los pendientes en las mejoras laborales en los estados donde el sector agropecuario cuenta con alta penetración de la agroindustria son grandes, en éstas últimas regiones lo son mucho más. Entre los retrocesos tenemos Baja California Sur, Chihuahua y 
Morelos principalmente, mientras que en los avances podemos destacar el de Baja California, Durango, Nayarit, Aguascalientes y Yucatán (Figura 6).

Figura 6. Porcentaje de mujeres con acceso a salud y otras prestaciones

Primer trimestre de 2008

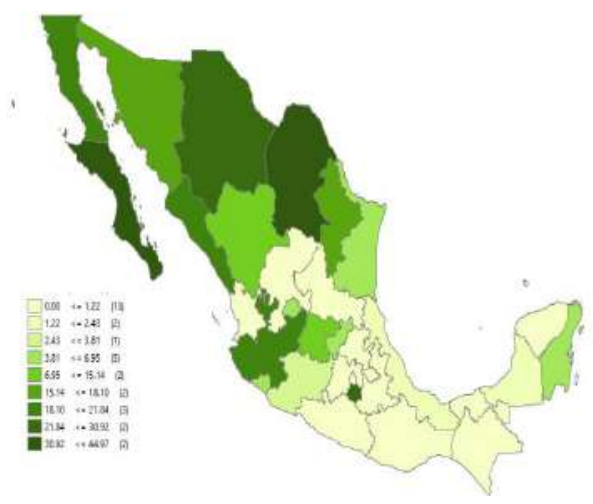

Primer trimestre de 2017

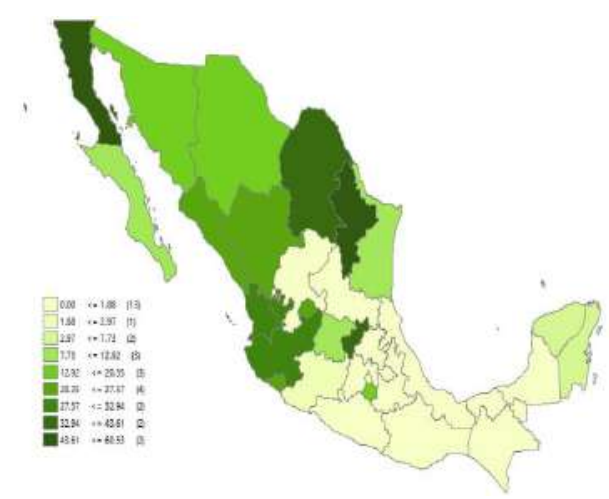

Fuente: elaboración propia con base en datos de la ENOE y el INEGI.

Por último, otro elemento de análisis es el de nivel de instrucción. Para 2008, a excepción de estados como Chihuahua, Nuevo León, Zacatecas y Michoacán, los estados del noroeste, noreste, centro norte y occidente del país presentaron las mayores tasas de ocupadas femenina con un nivel de instrucción mayor, mientras que, nuevamente, los estados del sur del país se vieron rezagados. Para 2017, la distribución se aprecia más marcada, pues se presentan retrocesos relativos en estados como San Luis Potosí, Veracruz, Estado de México, Tlaxcala y Tabasco; y avances en estados como Chihuahua y Nuevo León (Figura 7).

En este análisis se detecta que el factor de avance agroindustrial y su alta relación con el sector agropecuario es lo que ha marcado la diferencia, ya que ha generado actividades intensivas en el campo. Mientras que en los estados del norte se destaca este progreso, los del sur conservan aún técnicas productivas rezagadas - en la mayoría de los casos asociadas con la producción de autoconsumo- que no permiten la generación de un mayor valor agregado que se vea reflejado en salarios mayores. Estados como Puebla, Oaxaca y 
López-Martínez y Molina-Vargas: Análisis espacial de las condiciones laborales de la mujer...

Guerrero continúan teniendo una presencia importante de las actividades de autoconsumo, las cuales están asociadas a empleo no remunerado en la producción, principalmente de maíz (SAGARPA, 2013).

Figura 7. Porcentaje de mujeres con nivel de instrucción media-superior y superior Primer trimestre de 2008

Primer trimestre de 2017
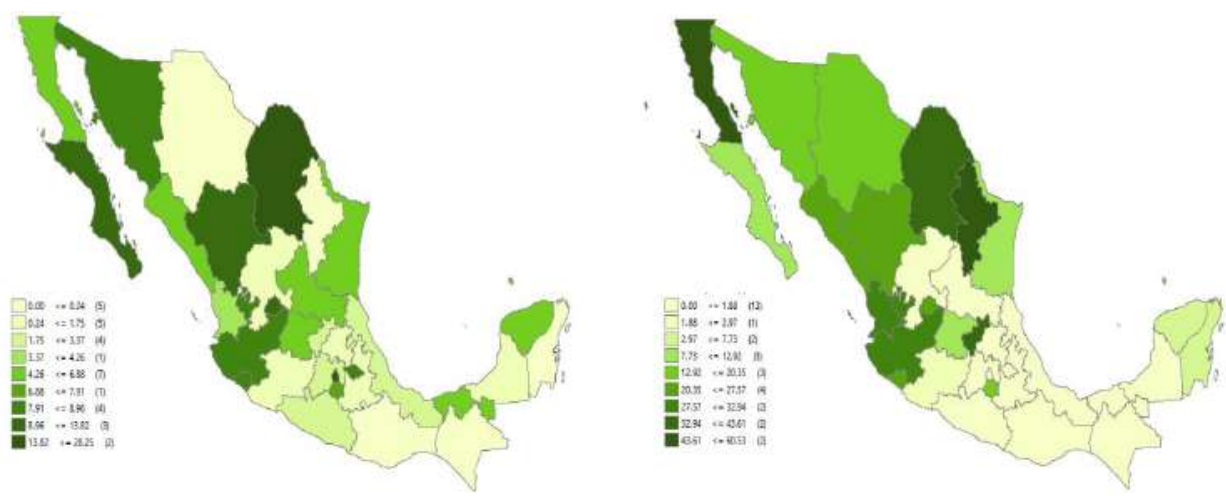

Fuente:elaboración propia con base en datos de la ENOE, INEGI.

Bajo el panorama descrito, toma mayor relevancia la inserción de políticas orientadas hacia el fomento de la intensificación de las ligas productivas, que van desde las actividades propiamente agropecuarias hasta las agroindustriales relacionadas con las exportaciones, ya que las actividades agroindustriales de exportación han sido las mayores generadoras de nuevos empleos en los años recientes (Molina \& López, 2017). Además, dichas actividades representan un vínculo esencial con el mercado interno en dos aspectos: la creación de empleos mejor remunerados (lo que generaría un incentivo a la demanda interna) y la generación de mayor valor agregado mediante el uso de mano de obra con mayor nivel de instrucción (lo que, en un marco de incentivos adecuados, impulsaría a sectores que ocupan personal con bajo nivel de instrucción a incrementar su nivel educativo). Dentro de todo este panorama, el papel de la mujer debe priorizarse, ya que los aumentos en la productividad de la mujer no se han reflejado en incrementos en sus remuneraciones (López \& Molina, 2018). Por lo tanto, las políticas deben estar orientadas a evitar este 
tipo de situaciones mediante la aplicación de principios de equidad de género, que permitan que las mujeres gocen de los beneficios de sus esfuerzos.

\section{IV. Índice de condiciones laborales}

Para medir los cambios de las condiciones laborales de las mujeres en el sector agropecuario por estados, analizados en el apartado anterior, se elabora un índice propio, utilizando datos de la ENOE del INEGI. Para su construcción, se tomaron en cuenta tres factores: la tasa de población femenina ocupada que percibe alguna remuneración (ponderada por segmentos de ingresos medidos en salarios mínimos), la tasa de población femenina ocupada que tiene acceso a salud y otras prestaciones, y la tasa de población femenina ocupada que no recibe ninguna remuneración. Así, tenemos que:

$$
I_{c l m}=\frac{\left[1+\left[\frac{\left(\frac{p}{5}+2 \frac{q}{5}+3 \frac{r}{5}+4 \frac{s}{5}+t\right)}{2}+\frac{u}{2}-v\right]\right]}{2},
$$

donde, $I_{c l m}$ es el índice de condiciones laborales de la mujer en el sector agropecuario, se tiene que $0 \leq I_{c l m} \leq 1 ; p$ representa el porcentaje de población femenina ocupada que percibe hasta un salario mínimo; $q$ es el porcentaje de población femenina ocupada que percibe más de un salario mínimo y hasta dos; $r$ es el porcentaje de población femenina ocupada que percibe más de dos salarios mínimos y hasta tres; $s$ es el porcentaje de población femenina ocupada que percibe más de tres salarios mínimos y hasta cinco; $t$ representa el porcentaje de población femenina ocupada que percibe más de cinco salarios mínimos; $u$ es el porcentaje de población femenina ocupada que tiene acceso a salud y otras prestaciones; y finalmente $v$ es el porcentaje de población femenina ocupada que no percibe ninguna remuneración. Todos los porcentajes están expresados en valores que se encuentran entre 0 y la unidad. $v$ presenta signo negativo, ya que entre mayor es la tasa de mujeres que no reciben remuneración alguna, menor es el valor del índice; no presenta ponderación con el fin de que, dado el escenario de que todas las mujeres ocupadas no percibieran ninguna remuneración, el numerador del índice sea 0 . 
López-Martínez y Molina-Vargas: Análisis espacial de las condiciones laborales de la mujer...

A fin de dar mayor peso en el índice a la tasa de mujeres ocupadas conforme perciben un ingreso cada vez mayor, cada segmento de mayor ingreso se pondera en mayor medida. Así, $p$ se pondera por un quinto, $q$ por dos quintos, etc. A su vez, el factor de mujeres remuneradas y el de mujeres que tienen acceso a salud y otras prestaciones se pondera por un medio cada uno, de tal manera que la suma de ambos, considerando que $v$ es excluyente (no puede existir el caso de que haya mujeres no remuneradas y remuneradas a la vez, o mujeres que no perciban ninguna remuneración y tengan acceso a salud y otras prestaciones laborales), da la unidad, la cual, al sumarse al 1 que le antecede, da como resultado 2 . A fin de estandarizar el resultado, este se divide entre 2, por lo que se da la unidad, el escenario ideal donde todas las mujeres ocupadas reciben una remuneración mayor a cinco salarios mínimos y tienen acceso a salud y otras prestaciones, cuyo índice de condiciones laborales es 1.

Se construye el índice para cada estado de la república para 2008 y para 2017. Como se ha señalado, el índice toma valor de 0 si el total de las mujeres ocupadas no reciben remuneración alguna y por supuesto no tienen acceso a salud y otras prestaciones, toma valor de 1 si el total de las mujeres ocupadas son remuneradas con más de cinco salarios mínimos y tienen acceso a salud y otras prestaciones. Por lo tanto, valores cercanos a la unidad reflejan condiciones laborales favorables, mientras que valores cercanos a 0 señalan condiciones laborales desfavorables para la mujer. Los resultados se muestran en la Figura 8.

A partir de la Figura 8, podemos observar tres cosas. Por un lado, algunos estados no presentaron cambios significativos, y en 2008 y 2017 continuaron presentando los mejores resultados; tales son los casos de los estados de Baja California, Baja California Sur, Sinaloa, Chihuahua y Sonora, cuyos índices de condiciones laborales para las mujeres se encuentran entre 0,59 y 0,74 . Por otro lado, tenemos los estados que tampoco presentaron cambios importantes, pero que se encuentran con índices de condiciones laborales muy bajos, es decir, que fluctúan entre 0,06 y 0,21; estos son los casos de Guerrero, Oaxaca y Chiapas. Finalmente, podemos ver que hay estados que presentan importantes cambios positivos; destacan aquí Querétaro, Aguascalientes, Estado de México, Guanajuato Coahuila y Nuevo León, cuyos índices se incrementaron entre 0,38 (en el caso, por ejemplo, de Querétaro, que pasó 
de 0,24 en 2008 a 0,62 en 2017) y 0,18 (en el caso de Nuevo León, que pasó de un índice de 0,42 en 2008 a 0,6 en 2017). Resulta importante señalar que los estados que han presentado una mejoría notoria en el índice de condiciones laborales son estados cuya industrialización ha sido especialmente relevante en los recientes años, lo que sugiere la existencia de un arrastre del sector industrial al sector agropecuario. Por último, es de destacar el retroceso que presentó la Ciudad de México, cuyo índice pasó de 0,4 en 2008 a 0,06 en 2017.

Figura 8. Índice de condiciones laborales de la mujer en el sector agropecuario

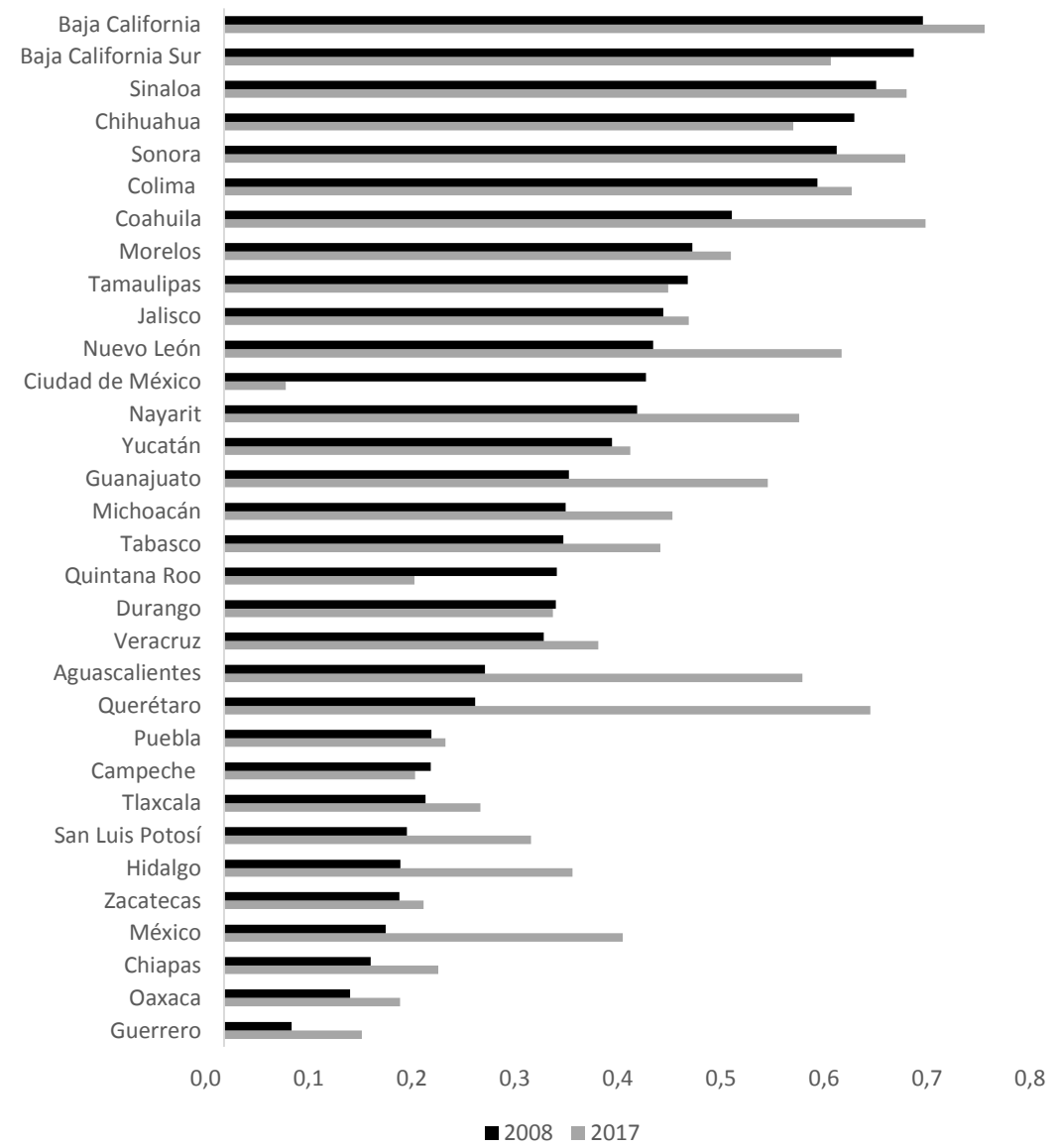

Fuente: elaboración propia con base en datos de la ENOE, INEGI. 
López-Martínez y Molina-Vargas: Análisis espacial de las condiciones laborales de la mujer...

El índice de condiciones laborales muestra las diferencias regionales existentes en el sector agropecuario en México en cuanto a salarios, inclusión a seguridad social y otras prestaciones laborales. Como resultado del desarrollo desigual, por un lado, se encuentran los estados del noroeste y noreste ligados al mercado internacional y, por otro lado, los estados del suroeste y sureste que continúan rezagados. La determinación de las características de las regiones ofrece la oportunidad de reconocer aquellas entidades federativas donde resulta importante el diseño y la implementación de políticas que ayuden a mejorar las condiciones de la mujer en este campo.

\section{Conclusiones}

Si bien, de 2008 a 2017, la participación del producto agropecuario dentro del producto total fue, en promedio, de tan solo el $32 \%$, a lo largo del periodo, el PIB agropecuario acumuló un crecimiento del 15,45\%, mientras que la ocupación femenina disminuyó en $3 \%$. Esto quiere decir que, en el sector agropecuario, la ley de Okun no se cumple para el caso femenino.

En cuanto a la estructura productiva del sector, poco más de la mitad de la producción agropecuaria se concentró en ocho estados de la república: Jalisco, Sinaloa, Michoacán, Veracruz, Sonora, Chihuahua, Guanajuato y Puebla. Por otro lado, las diferencias regionales de participación laboral de la mujer en el sector son grandes, pues el mercado laboral agropecuario por estados es bastante segmentado: en seis estados se concentró la mitad de la ocupación femenina: Puebla, Oaxaca, Guerrero, Jalisco, Guanajuato y Michoacán. De estos, los tres primeros son los que más absorben el trabajo femenino; sin embargo, se trata de ocupación no remunerada. Esto revela que en dichos estados existe una disociación entre producción agropecuaria y ocupación femenina, pues mientras la contribución al PIB agropecuario es baja, la ocupación femenina no remunerada es relativamente alta.

Por otra parte, en el sector agropecuario, la ocupación femenina con menores remuneraciones se encuentra en los estados del centro sur, suroeste y sureste, donde, a lo largo del periodo de estudio, continúan presentándose porcentajes muy bajos de mujeres que perciben un ingreso de más de cinco salarios mínimos. En contraste, hubo mejoras en los estados del noroeste, 
como Sonora, Chihuahua y Sinaloa, y en el noreste, como en Tamaulipas. Respecto al acceso a salud y a otras prestaciones, se observa una clara correlación con la distribución de las mujeres con mayor remuneración, por lo que en los estados del noroeste se observan mayores porcentajes de mujeres que cuentan con estos derechos laborales.

El aumento de mujeres con niveles de educación medio-superior y superior ha repercutido en su paulatina incorporación al mercado laboral del sector agropecuario; una de las explicaciones se encuentra en que los otros sectores de la actividad económica ya están saturados. Sin embargo, el aumento del nivel de educación de las mujeres tampoco influye de manera directa en su ingreso. A excepción de estados como Chihuahua, Nuevo León, Zacatecas y Michoacán, todos los estados del noroeste, noreste, centro norte y occidente del país presentaron las mayores tasas de ocupación femenina con un nivel de instrucción mayor, mientras que, nuevamente, los estados del sur del país se vieron rezagados. Una explicación a esto la podemos encontrar en la literatura empírica (Molina \& López, 2017), donde se destaca que el factor de avance agroindustrial y su alta relación con el sector agropecuario es lo que ha marcado la diferencia; es decir, un mayor valor agregado a los productos agropecuarios ha generado actividades intensivas en el campo. En los estados del norte se destaca esta actividad, pero los del sur conservan aún técnicas productivas rezagadas, en la mayoría de los casos asociadas con la producción de autoconsumo y de empleo no remunerado, que no permite la generación de un mayor valor agregado que se traslade a salarios mayores; estos son los casos como Puebla, Oaxaca y Guerrero.

Por último, el índice de condiciones laborales que se realiza muestra que los estados del sur, Guerrero, Oaxaca y Chiapas, presentan condiciones laborales muy bajas para las mujeres, con índices menores a 0,21 , mientras que estados del noroeste, Baja California, Baja California Sur, Chihuahua, Sinaloa y Sonora, aunque no presentan cambios significativos, continúan presentando los mejores resultados, con índices que se encuentran entre 0,59 y 0,74. Dentro de los estados que mejoraron las condiciones laborales se encuentran Querétaro, Aguascalientes, Estado de México, Guanajuato Coahuila y Nuevo León, los cuales tienen una alta industrialización. Esto sugiere la existencia de un efecto spillover sobre el sector agropecuario. 
López-Martínez y Molina-Vargas: Análisis espacial de las condiciones laborales de la mujer...

\section{Referencias}

Aranda, P. \& Castro, M. (2016). El campo de la agroindustria en el noroeste de México y la salud de sus jornaleras: una propuesta de estudio. Revista Salud Colectiva, 12(1), 55-70. doi: 10.18294/sc.2016.878

Arriagada, I. (1990). La participación desigual de la mujer en el mundo del trabajo. Revista de la CEPAL, 40, 87-104. Recuperado de http://repositorio.cepal.org/bitstream/handle/11362/11764/04008710 4_es.pdf?sequence $=1$

Becker, G. (1981). A Treatise on the Family. Cambridge: Harvard University Press.

Caro, P. (2012). Magnitud y características de la participación laboral en el empleo temporal agrícola en Chile. Revista de Estudios Transfronterizos, 12(2), 51-83. Recuperado de http://www.redalyc.org/ articulo.oa?id=337929288003

Carrillo, J. \& Hernández, A. (2014). Mujeres fronterizas en la industria maquiladora. México: Colef.

Castaño, Cecilia (1999). Economía y género. Política y Sociedad, 32, 23-42.

Cervini, H. (2000). Recesión y empleo femenino. Economía Teoría y Práctica, $12,55-92$.

Cordourier, G. \& Gómez, A. (2004). La evolución de la participación laboral de las mujeres en la industria: una visión de largo plazo. Economía Mexicana. Nueva Época, 13(1), 63-104. Recuperado de http://www.redalyc.org/articulo.oa?id=32313104

Craig, C., Garnsey, E. \& Rubery, J. (1985). Labour market segmentation and women's employment: a case-study from United Kingdom. International Labour Review, 124(3), 267-280.

De Garay, A. (2013). Igualdad de género en la Universidad Autónoma Metropolitana: un espejismo. Revista de Estudios de Género, La Ventana, 4 (38) 277-330. 
Doeringer, P. \& Piore, M. (1971). Internal Labor Markets and Manpower Adjustment. New York: DC Heath and Company.

Elborgh, K., Newiak, M., Kochhar, K., Fabrizio, S., Kpodar, K., Wingender, P., Clements, B. \& Schwartz, G. (2013). Las mujeres, el trabajo y la economía: beneficios macroeconómicos de la equidad de género. Recuperado de http://www.igualdadenlaempresa.es/ enlaces/webgrafia/docs/Las_mujeres_el_trabajo_y-FMI.pdf

Ferber, M. \& Nelson, J. (1993). Beyond Economic Man, feminist theory and economics. Chicago: University Chicago Press.

Flores, L. \& Salas, I. (2015). Las brechas de género en la calidad del empleo en México. Una valoración basada en modelos de lógica difusa. Análisis Económico, 30(75), 89-112. Recuperado de http://www.redalyc.org/articulo.oa?id=41343702004

Fouquet, A. (2005). Diferencias regionales en México: una herencia geográfica y política. En N. Guzmán (Comp.), Sociedad y desarrollo en México (2da. ed.) (pp. 385-590). Monterrey: Ediciones Regiomontanas.

Gabayet, L. \& Lailson, S. (1990). Mundo laboral, mundo doméstico: obreras de la industria manufacturera de Guadalajara. Estudios Sociológicos, 8(24), 547-570.

García, L. (2015). El papel de la mujer en el desarrollo de México. Revista Contribuciones a las Ciencias Sociales, 27. Recuperado de: http:// www.eumed.net/rev/cccss/2015/01/mujer.html

Guzmán, F. (2004). Inequidad por género en el sector terciario. Revista Economia Informa, 324, 86-94.

Hernández, J. (2014). Condiciones de trabajo e ingreso en la agricultura intensiva mexicana. Análisis Económico, 2971, 137-160. Recuperado de: http://www.redalyc.org/articulo.oa?id=41333722007

Lahoz, D. (2011). Mujeres campesinas y su papel en el sistema alimentario en México. México D.F.: Oxfam México. 
López-Martínez y Molina-Vargas: Análisis espacial de las condiciones laborales de la mujer...

López, J. \& Molina, A. (2018). La situación laboral de la mujer en el sector agropecuario en México, 2008-2016. Análisis Económico, 33(83), $97-$ 123. doi: 10.24275/uam/azc/dcsh/ae/2018v33n83/lopez

Martínez, C., Quintero, C., \& Viianto, L. (2015). Discriminación de género en redes laborales. Ensayos Revista de Economia, 34(2), 1-34.

Mendoza, M., Cardero, M. \& Ortiz, A. (2017). Algunos hechos estilizados y explicativos sobre el diferencial y la discriminación salarial por sexo en México, 1987-2015. Investigación Económica, 76(301), 103-135.

Moctezuma, D., Narro, J. \& Orozco, L. (2014). La Mujer en México: inequidad, pobreza y violencia. Revista Mexicana de Ciencias Politicas y Sociales, 59(220), 117-146.

Molina, A. \& López, J. (2017). El impacto de las exportaciones no petroleras sobre el empleo en México, 1995-2016. Tiempo Económico, 12(35), 4965. doi: 10.24275/uam/azc/dcsh/te/2018v12n35.molina

Noriega, F. (2010). Microfundamentos para la economía de la mujer. Economía y Sociedad, 14(25), 69-89.

Piore, M. (1972). Notes for a theory of labor market stratification (Working Paper No, 95). Recuperado de Massachusetts Institute of Technology: https://dspace.mit.edu/handle/1721.1/64001

Rodríguez, J. (2003). La economía laboral en el periodo clásico de la historia del pensamiento económico (Tesis Doctoral, Universidad de Valladolid, España). Recuperado de http://www.cervantesvirtual.com/obra/laeconomia-laboral-en-el-periodo-clasico-de-la-historia-del-pensamientoeconomico-0/

SAGARPA (2013). Monitor Agroeconómico e Indicadores de la Agroindustria. México: Subsecretaría de Alimentación y Competitividad.

SAGARPA (2017). Atlas Agroalimentario. México: SIAP.

Solow, Robert (1993). Feminist Theory, Women's Experience, and Economics. En M. Ferber \& J. Nelson (Comps.), Beyond Economic Man: 
Feminist Theory and Economics (pp. 153-158). Chicago: University of Chicago Press.

Valenciano, J., Capobianco, M. \& Toril, J. (2017). Vulnerabilidad laboral de la mujer rural latinoamericana. Nóesis. Revista de Ciencias Sociales y Humanidades, 26(52), 130-151, doi: 10.20983/noesis.2017.2.6

Villegas, C. (2012). Economía de género y teoría del crecimiento económico: la relación entre mujeres-madre y agentes consumidores. Revista Economía, 33, 65-83. 